Marie-Pascale Halary, « Le miroir de Guillaume de Lorris à l'école de la charité », dans Les Écoles de pensée du XII siècle et la littérature romane (oc et oül), dir. Valérie Fasseur et Jean-René Valette, Turnhout, Brepols, « Bibliothèque d'histoire culturelle du Moyen Âge », 2016, p. 279-299.

DOI : 10.1484/M.BHCMA EB.5.109800

\title{
Le miroir de Guillaume de Lorris à l'école de la charité*
}

S'il est un Roman de la Rose que la critique aime à rapprocher des écoles de pensée, c'est celui de Jean de Meun. Des travaux fondateurs ont repéré dans cette œuvre, composée pendant le dernier tiers du XIII ${ }^{\mathrm{e}}$ siècle, des sources ou des influences qui, depuis Alain de Lille $^{1}$ jusqu'à la scolastique ${ }^{2}$, témoignent de cette culture scolaire qui est celle de l'auteur ${ }^{3}$. Le « premier » Roman de la Rose, en revanche, celui qui est attribué à Guillaume de Lorris ${ }^{4}$ et qui a vraisemblablement été composé autour de 1230, semble moins se prêter à une telle confrontation: si de nombreux intertextes ont pu être identifiés (poésie antique, lyrique courtoise, arts poétiques, etc..$^{5}$ ), il paraît moins pertinent de rapporter ce récit courtois à ce que l'on appelle une « école de pensée ». On a ainsi fréquemment exprimé le rapport entre le texte de Guillaume et sa continuation en termes d'opposition : cette divergence dans la nature des sources utilisées contribuerait à expliquer les différences dans la conception de l'amour ainsi

\footnotetext{
* Nous adressons nos plus sincères remerciements à Éléonore Andrieu et Jean-René Valette qui nous ont permis de corriger certaines des imperfections de ce travail.

${ }^{1}$ Voir en particulier Ernest Langlois, Origines et sources du Roman de la Rose, Paris, Ernest Thorin, 1890, p. 149 sq.

${ }^{2}$ Gérard Paré, Le Roman de la Rose et la scolastique courtoise, Paris, Vrin, 1941.

3 «L'œuvre de Jean apparaît comme le miroir, moins d'un génie individuel, que du génie d'une époque entière » (Edmond Faral, «Le Roman de la Rose et la pensée française au XIII ${ }^{\mathrm{e}}$ siècle », Revue des deux mondes, 1926, 9, t. 35, p. 452).

${ }^{4}$ Guillaume de Lorris, Le Roman de la Rose, éd. Félix Lecoy, Paris, Champion, « Classiques français du Moyen Âge », 1983, t. 1.

${ }^{5}$ Sur le rapport avec les textes de langue latine, voir notamment la première partie de l'ouvrage d'Ernest Langlois, Origines et sources du Roman de la Rose, op. cit. ainsi qu'Edmond Faral, "Le Roman de la Rose et la pensée française au XIII ${ }^{\mathrm{e}}$ siècle », art. cit., p. 431-437.
} 
que dans la représentation de la figure auctoriale ${ }^{6}$. D'un côté, l'œuvre d'un poète; de l'autre celle d'un philosophe - selon une distinction d'Erich Köhler'.

Nous voudrions toutefois présenter ici ce qui n'est qu'une hypothèse : est-ce que certains textes cisterciens, plus proches de ce qui serait une "école de pensée ${ }^{8}$, ne pourraient pas éclairer, dans le récit de Guillaume de Lorris, l'épisode du miroir ?

\section{Les cisterciens et Guillaume : une approche possible ?}

Dans ce passage fort connu, la fontaine dans laquelle Narcisse trouva la mort est décrite comme un extraordinaire dispositif spéculaire : deux cristaux disposés au fond sont éclairés par les rayons du soleil qui colorent l'eau de mille teintes. Alors, «ausi con li mireors » (v. 1553) indique le narrateur, ces cristaux réfléchissent chacun la moitié du verger, « tot a orne» (v. 1550). Cette vision constitue une étape importante dans le parcours de l'Amant : c'est précisément dans ces cristaux que le jeune homme aperçoit pour la première fois le reflet des « rosiers chargiez de roses » (v. 1614).

Cet épisode célèbre a fait l'objet de très nombreuses analyses : on y a vu un passage central pour des questions génériques ${ }^{9}$ et intertextuelles ${ }^{10}$; on y a vu aussi un rappel du mythe platonicien de la caverne ${ }^{11}$. Un certain nombre de lectures ont notamment cherché, selon des approches souvent très différentes, à prendre en compte le contexte historique : l'image du

${ }^{6}$ Qu'il s'agisse de deux « auteurs » différents ayant chacun une identité historique propre ou que Jean de Meun (voire même un autre) se soit dissimulé derrière le masque fictionnel de Guillaume de Lorris. Sur cette dernière hypothèse, $c f$. Roger Dragonetti, "Pygmalion ou les pièges de la fiction dans le Roman de la Rose », dans La Musique et les lettres. Études de littérature médiévale, Genève, Droz, «Publications romanes et françaises », 1986, p. 345-367 ainsi que id., Le Mirage des sources. L'art du faux dans le roman médiéval, Paris, Seuil, 1987, p. 200 sq., Fabienne Pomel, « La fonction-auteur dans le Roman de la Rose de Jean de Meun : le double jeu de la consécration et de l'esquive », dans Nicole Jacques-Lefèvre (dir.), Une histoire de la «fonction-auteur » est-elle possible ?, Saint-Étienne, Publications de l'Université de Saint-Étienne, 2001, p. 89-106, Luciano Rossi, « De Jean Chopinel à Durante : la série Roman de la Rose-Fiore », dans Catherine Bel et Herman Braet (dir.), De la Rose. Texte, Image, Fortune, Louvain/Paris/Dudley, Peeters, 2006, p. 273-298.

${ }^{7}$ Erich Köhler, « Narcisse, la Fontaine d'Amour et Guillaume de Lorris », Journal des savants, 1963, $\mathrm{n}^{\circ} 2$, p. 95 .

${ }^{8}$ Comme l'explique notamment Jacques Paul, si à Cîteaux il n'y a pas d'école stricto sensu (la formation «scolaire», dans des écoles urbaines parfois, précède la vie monastique), la valeur métaphorique du terme est pleinement actualisée en raison de l'intensité de la vie intellectuelle : «il s'agit [...] d'une communauté de pensée et d'un courant d'échanges entre un savant éminent et des moines qui veulent bien se reconnaître pour ses disciples. À vrai dire, en dehors de l'éducation des jeunes moines, n'était-ce pas le seul sens que l'on puisse donner au terme d'école monastique ?» (Jacques Paul, L'Église et la culture en Occident (IX-XII siècle), t. 2, L'Éveil évangélique et les mentalités religieuses, Paris, PUF, 1986, p. 502).

${ }^{9}$ Par exemple Joan Kessler, « La quête amoureuse et poétique : la fontaine de Narcisse dans le Roman de la Rose », Romanic Review, 1982, n 73/2, p. 133-146.

${ }^{10}$ Signalons surtout l'article d'Emmanuèle Baumgartner qui compare plusieurs reprises médiévales du récit ovidien : « Narcisse à la fontaine : du "conte" à l'"exemple" », Cahiers de recherches médiévales et humanistes [en ligne], 2002, nº 9, mis en ligne le 5 janvier 2007, consulté le 6 août 2012.

${ }^{11}$ Jean Frappier, " Variations sur le thème du miroir, de Bernard de Ventadour à Maurice Scève », Cahiers de l'Association internationale des études françaises, 1959, $\mathrm{n}^{\circ} 11, \mathrm{p} .134-158$. 
miroir a ainsi pu être rapprochée de certaines «idées superstitieuses et magiques » ${ }^{12}$ qui auraient été fort répandues au Moyen Âge; elle a été également mise en relation avec la théorie médiévale du fantasme ${ }^{13}$, avec les différents niveaux d'interprétation de l'exégèse allégorique $^{14}$ comme avec plusieurs textes courtois ${ }^{15}$. Or, comme l'a montré Bernard Lahire dans le champ des sciences sociales ${ }^{16}$, le contexte d'un objet d'étude n'est pas unique et il ne s'impose pas à la manière d'une évidence; il s'agit avant tout de la construction d'un chercheur. Tout l'enjeu est alors d'essayer d'articuler la sélection de ce contexte au « champ de validité » des conclusions de l'observation. En d'autres termes, ne pourrait-on pas aussi pour examiner la fontaine de Guillaume de Lorris partir, non de la réalité historique du miroir médiéval, mais d'un de ses emplois comme image dans un discours ${ }^{17}$ ?

En effet, veoir "parmei mirors $»^{18}$ est une des manières dont la langue romane rend compte de la formule paulinienne à laquelle la référence au speculum ne manque pas d'être associée $^{19}$ :

Videmus nunc per speculum in aenigmate : tunc autem facie ad faciem. Nunc cognosco ex parte : tunc autem congnoscam sicut et cognitus sum. (I Cor 13,12)

Sans que cette proposition soit exclusive d'aucune des autres pistes qui ont été évoquées, nous voudrions donc examiner en quoi cette référence (dont la nature de la relation avec la fiction reste à préciser) peut éclairer cet épisode du Roman de la Rose. Ce passage de l’Épître aux Corinthiens, on le sait, fait au Moyen Âge l'objet de nombreux commentaires qui ont été particulièrement étudiés par Einar $\mathrm{Ma} \mathrm{r}$ Jo nsson ${ }^{20}$. Celui-ci montre surtout que la

${ }^{12}$ Erich Köhler, « Narcisse, la Fontaine d'Amour et Guillaume de Lorris », art. cit., p. 98.

${ }^{13}$ Giorgio Agamben, Stanze. Parole et fantasme dans la culture occidentale, trad. Yves Hersant, Paris, Christian Bourgeois éditeur, 1981 [1977], p. 105 sq. Cette piste a ensuite été reprise par Henri ReyFlaud, "Le miroir de Narcisse du Roman de la Rose. Contribution à une anthropologie littéraire », dans Sources et fontaines du Moyen Âge à l'âge baroque, Actes du colloque tenu à l'Université Paul Valéry-Montpellier III les 28, 29 et 30 novembre 1996, Paris, Champion, 1998, p. 129-137.

${ }^{14}$ Charles Méla, « Le miroir périlleux ou l'alchimie de la rose », Europe, octobre 1983, n 654 , p. 77 $s q$.

${ }^{15}$ Outre les références déjà indiquées, voir David F. Hult, "The allegorical Fountain: Narcissus in the Roman de la Rose", Romanic Review, 1981, n 72/2, p. 130.

16 Bernard Lahire, «La variation des contextes dans les sciences sociales. Remarques épistémologiques », Annales. Histoire, Sciences sociales, 1996, n² 2, p. 381-407.

${ }^{17}$ Nous nous proposons donc de partir, non pas de la pensée médiévale du miroir ou des pratiques liées à cet objet, mais de certains discours dans lesquels la référence au miroir, comme dans notre récit, est une analogie.

${ }^{18}$ La Traduction en prose française du XII siècle des Sermones in Cantica de saint Bernard, éd. Stewart Gregory, Amsterdam, Rodopi, «Faux titre », 1994, sermon 31, p. 210 par exemple: "nos veons or parmei mirors et en figure, ne mie encor face a face $»$.

${ }^{19}$ «Le mot speculum [...] renvoie presque automatiquement le lecteur médiéval au célèbre verset 13,12 de la Première épître aux Corinthiens [...], d'où les auteurs patristiques ont extrapolé une théorie de la connaissance des réalités supérieures de type platonicien » (Jean-Yves Tilliette, Des mots à la parole. Une lecture de la Poetria nova de Geoffroy de Vinsauf, Genève, Droz, 2000, p. 62).

${ }^{20}$ Einar Ma r Jo nsson, Le Miroir. Naissance d'une genre littéraire, Paris, Les Belles Lettres, « Histoire », 1995. Pour les interprétations de cette formule au XII ${ }^{\mathrm{e}}$ siècle, voir aussi Robert Javelet, Image et ressemblance au XII siècle, de saint Anselme à Alain de Lille, Paris, Letouzé et Ané, 1967, t. 1 , p. $376-390$. 
métaphore du miroir se construit dans une histoire; celle-ci remonte au moins à Platon ${ }^{21}$ et elle se prolonge bien après saint Paul : de l'Antiquité tardive au cœur de la période médiévale, nombreuses sont les interprétations de la formule paulinienne. Il semble dès lors plus juste de s'intéresser à une des manières dont ce passage peut être reçu pendant le Moyen Âge central : selon cette perspective, nous nous concentrerons donc sur quelques auteurs habituellement rattachés à l'« école » cistercienne.

Différentes raisons expliquent ce choix. Il paraît en effet difficile de mener une enquête approfondie sur la présence de la métaphore du miroir dans les écrits monastiques du $\mathrm{XII}^{\mathrm{e}}$ siècle, tant il est vrai que cette image se retrouve partout. Il semble toutefois que les références au speculum paulinien sont plus fréquentes encore chez Bernard de Clairvaux et ses compagnons $^{22}$. Einar Ma r Jo nsson, dont l'étude s'arrête au seuil du XII ${ }^{\mathrm{e}}$ siècle, note en ce sens que c'est précisément dans les milieux cisterciens que le Miroir des vierges, ouvrage fondamental pour l'évolution du symbolisme du miroir, a été d'abord diffusé23. Il apparaît en outre que, chez ces spirituels, cette métaphore est particulièrement sollicitée quand il s'agit de décrire le parcours mystique ${ }^{24}$. Autrement dit, avec cette $"$ schola caritatis ${ }^{25}$ que constitue 1 '« école » cistercienne, nous nous trouvons face à des discours qui ordonnent la double vision (per speculum in aenigmate d'une part, facie ad faciem d'autre part) à la question de l'amour : cette relation entre voir et aimer ne manque pas d'intérêt pour qui désire se concentrer sur le Roman de la Rose. Il s'agira donc d'examiner conjointement le texte en langue romane et les constructions intellectuelles déterminées par la formule paulinienne dans certains écrits cisterciens.

Une telle enquête, on le voit, ne vise pas a priori à repérer des « sources » ou des « influences» du Roman et elle ne se veut nullement exclusive des études qui ont été

${ }^{21}$ Sur ce point, voir aussi Norbert Hugedé, La Métaphore du miroir dans les Épittres de saint Paul aux Corinthiens, Neuchâtel/Paris, Delachaux et Niestlé, 1957.

${ }^{22}$ De manière révélatrice, cette référence est absente du De tribus diebus d'Hugues de Saint-Victor (éd. Dominique Poirel, dans Opera Hugonis de sancto Victore, t. 2, Turnhout, Brepols, "Corpus christianorum. Continuatio mediaevalis », 2002) qui se concentre pourtant sur les rapports entre les visibilia et les invisibilia (mais en recourant plutôt à la fameuse image du livre). S'agissant de cet auteur, il ne faudrait toutefois pas généraliser : on retrouve par exemple la formule paulinienne dans le commentaire de la Hiérarchie céleste (Commentariorum in Hierarchiam Coelestem S. Dionysii Areopagitce, PL 175).

${ }^{23}$ Einar Ma r Jo nsson, Le Miroir..., op. cit., p. 203 sq.

${ }^{24}$ Einar Ma $\mathrm{r}$ Jo nsson indique que cette innovation, l'association du symbolisme du miroir et du symbolisme nuptial du Cantique des cantiques, est le fait du Miroir des vierges (ibid., p. 171 sq.).

${ }_{25}$ «Haec est specialis caritatis scola; hic ejus studia exercentur disputationes agitantur, solutiones non ratiocinationibus tantum quantum ratione et ipsa rerum veritate et experientia terminantur » (Guillaume de Saint-Thierry, De natura et dignitate amoris, dans Deux traités de l'amour de Dieu: De la contemplation de Dieu, De la nature et de la dignité de l'amour, éd. et trad. Marie-Madeleine Davy, Paris, Vrin, 1953, 31, p. 108-109 : «Voilà [dans le monastère] l'école spéciale de charité. Là se déroulent les exercices de son apprentissage; des discussions s'y agitent. Les solutions ne sont pas tant données par des raisonnements que par la raison, par la vérité même des choses et l'expérience »). Sur le sens de cette expression de schola caritatis et sur les rapports entre l'école du Christ, dans le monastère, et l'école du siècle, $c f$. Étienne Gilson, La Théologie mystique de saint Bernard, Paris, Vrin, 1947, p. $78 s q$. 
auparavant signalées. Simplement, il est peut-être possible de penser la confrontation entre les discours en d'autres termes également. La métaphore du miroir, en cela, serait plus qu'un topos: en diachronie, elle est successivement intégrée à des constructions spécifiques qui actualisent et développent telle ou telle de ses potentialités sémantiques. À ce titre, on pourrait considérer qu'elle est le noyau de différents discours dont l'historicité ne saurait être négligée. L'image du speculum dans le roman pourrait alors être envisagée comme un «indic[e] de contextualisation », comme un lieu d'affleurement du «contexte» dans le texte ${ }^{26}$. Précisément, Michel Foucault, après avoir fait table rase de la relation causale et d'une des formes que peut prendre celle-ci, la relation d'influence, propose d'examiner des énoncés hétérogènes et singuliers à partir de leur seule simultanéité ou de leur appartenance à une commune épistémè. Dès lors que deux discours sont (relativement) contemporains, ils sont, pourrions-nous dire, «compossibles »: ils sont déterminés par les mêmes conditions de possibilité, sans qu'il soit nécessaire d'établir entre eux un lien direct et "génétique ». Cette manière de penser le rapport entre des discours différents, démarche qui s'attache d'abord au repérage de ressemblances entre les faits discursifs, présente l'intérêt d'autoriser des rapprochements plus inattendus. Ainsi de la comparaison entre Guillaume de Lorris et les cisterciens $^{27}$.

On pourrait alors considérer les écrits des spirituels dont il est question ici comme des manifestations d'une sorte de discours possible ou de discours disponible au début du $\mathrm{XIII}^{\mathrm{e}}$ siècle : comment dire l'excellence d'une vision (courtoise) sinon en reprenant les images et les configurations discursives disponibles ? Comment signifier l'incomparable excellence de l'objet aimé sinon en soumettant sa contemplation à une observation dans un miroir ? Sous ce rapport, la lecture des mystiques peut sembler particulièrement féconde puisqu'il s'agit précisément pour ces auteurs de rendre compte de la plus haute des visions, de celle qui confine à l'indicible mais qui, pourtant, bien que dans une certaine mesure, se laisse saisir dans un espace dicible. Nous voudrions donc faire l'hypothèse qu'on peut peut-être recourir aux cisterciens pour envisager ce passage quelque peu énigmatique du Roman de la Rose selon la perspective des limites et des possibilités historiques du dire ${ }^{28}$.

\section{Le miroir de Guillaume : un haut lieu du discours courtois}

${ }^{26}$ Claire Badiou-Monferran, «Présentation. Le dialogue interdisciplinaire: un "conte de peau d'âne" ? ", dans Claire Badiou-Monferran (dir.), Il était une fois l'interdisciplinarité. Approches discursives des Contes de Perrault, Louvain-la-Neuve, Academia-Bruylant, "Au cœur des textes », 2010 , p. 55.

${ }^{27}$ Sur cette démarche, nous nous permettons de renvoyer aux développements que nous avons présentés dans « Michel Foucault et l'étude du contexte médiéval des œuvres littéraires : un discours de la méthode ?», dans Damien Boquet, Blaise Dufal et Pauline Labey (dir.), Une histoire au présent. Michel Foucault à l'épreuve, actes de la journée d'étude organisée à l'EHESS le 27 mars 2010, à paraître aux Presses Universitaires de Rennes.

${ }^{28}$ Ce passage est très librement inspiré par une réflexion stimulante de Roger Chartier: «ce qu'il importe de comprendre n'est plus les audaces du pensé mais bien plus les limites du pensable » (Roger Chartier, Au bord de la falaise. L'histoire entre certitudes et inquiétude, Paris, Albin Michel, «Histoire », 1998, p. 37). 


\section{VISION - Le miroir comme image de la contemplation indirecte}

Le speculum paulinien tel qu'il est interprété par les cisterciens et nombre de leurs prédécesseurs pourrait tout d'abord permettre de mieux comprendre le dispositif décrit dans le Roman de la Rose. On a souvent préféré comparer celui-ci à certains miroirs présents dans la lyrique mais, bien qu'il soit incontestable que toutes ces réalisations ressortissent à une même tradition, le rapprochement avec les textes mystiques peut être tout aussi fécond. Ainsi, chez Bernard de Ventadour, auteur souvent cité29, cette référence est une analogie dont le comparé est clairement identifié : le miroir que contemple l'amant, ce sont les yeux de sa dame ${ }^{30}$. Dans cette relation spéculaire, il y a donc d'une part le spectateur-amant et, d'autre part, la Dame dont les yeux agissent comme un miroir. Chez Guillaume de Lorris, l'objet aimé et la fontaine-miroir ne sont pas identifiés : l'amant contemple les «.II. pierres de cristal» (v. 1536) et ces cristaux eux-mêmes reflètent le verger et ses roses. Ce serait précisément parce que le miroir est un relais, un instrument de médiation, qu'il rappelle plus le speculum évoqué par les spirituels ${ }^{31}$. Comme tant d'autres, il arrive à Guillaume de Saint-Thierry de comparer les Écritures à un miroir ${ }^{32}$. Et Marie-Madeleine Davy de commenter :

Qu'est-ce que percevoir par un miroir ? C'est connaître quelque chose non pas en le voyant directement et immédiatement, mais en le voyant réfléchi par un miroir, qui est ici

${ }^{29}$ C'est sur cet auteur que Jean Frappier s'appuie essentiellement dans son étude « Variations sur le thème du miroir... », art. cit., p. 151 sq. Pour une autre comparaison avec le troubadour, voir Zuzanna Marcinkowska, «La prison amoureuse de Guillaume de Lorris », Médiévales, 1988, n 14, p. 103-112. ${ }^{30}$ " Anc non agui de me poder / Ni no fui meus de l'or'en sai / Que. m laisset en sos olhs vezer / En un miralh que mout me plai. / Miralhs, pus me mirei en te, / M'an mort li sospir de preon, / C'aissi. m perdei com perdet se / Lo bels Narcissus en la fon »: «Plus jamais je n'eus de pouvoir sur moi, plus jamais je ne m'appartins, depuis l'heure où dans ses yeux elle me laissa regarder en un miroir qui tant me plait. Miroir, depuis qu'en toi je me suis miré, les profonds soupirs m'ont tué et je me suis perdu comme se perdit le beau Narcisse dans la fontaine » (Bernard de Ventadour, éd. C. Appel (Halle, 1915), chanson XLIII, v. 17-24, cité et traduit par Jean Frappier, "Variations sur le thème du miroir... », art. cit., p. 152-153).

${ }^{31}$ Ce statut de relais accordé à un reflet dans un miroir n'est pas absent non plus de la littérature courtoise, ce dont témoigne notamment Le Lai de l'ombre de Jean Renart, éd. Félix Lecoy, Paris, Champion, "Classiques français du Moyen Âge », 1979: tandis que la maîtrise des codes et de la rhétorique de la courtoisie par le chevalier est insuffisante pour que celui-ci gagne l'amour de sa dame, il finit par triompher en offrant un anneau au reflet de celle qu'il aime. Cette ombre, qui apparait au fond d'un puits, peut être envisagée comme un double inaccessible et idéalisé du personnage féminin et il est doté d'une relative autonomie par rapport à l' « original». Dans ce récit, le geste du chevalier, qui constitue une «bele cortoisie» (v. 920), passe donc bien par la médiation d'une figuration de la dame. Mais au-delà de ces similitudes, nous voudrions montrer que la présence et les caractéristiques des cristaux chez Guillaume de Lorris déterminent un fonctionnement spécifique, plus proche, dans une certaine mesure, des constructions élaborées autour de la formule paulinienne.

${ }^{32}$ Par exemple Guillaume de Saint-Thierry, Enigma fidei, dans Guillaume de Saint-Thierry, Deux traités sur la foi. Le Miroir de la foi, l'Énigme de la foi, éd. et trad. Marie-Madeleine Davy, Paris, Vrin, "Bibliothèque des textes philosophiques », 1959, 3, p. 92-94. Cf. Einar Ma r Jo nsson, «Le sens du titre Speculum aux $\mathrm{XII}^{\mathrm{e}}$ et $\mathrm{XIII}^{\mathrm{e}}$ siècles et son utilisation par Vincent de Beauvais », dans Monique Paulmier-Foucart, Serge Lusignan et Alain Nadeau (dir.), Vincent de Beauvais, intentions et réceptions d'une æuvre encyclopédique au Moyen Âge, Saint-Laurent/Paris, Maison Bellarmin/Vrin, 1990, p. 19 sq. 
moyen, intermédiaire. Connaître Dieu dans le miroir, c'est le connaître par autre chose que lui et qui pourtant le reflète ${ }^{33}$.

Même si dans le Roman de la Rose il ne s'agit en aucun cas de la contemplation divine, il semble qu'on puisse repérer un mode de vision semblable ${ }^{34}$ : il s'agirait dans les deux cas de ce qu'on appelle la vision catoptrique ${ }^{35}$.

Cette interprétation pourrait contribuer à expliquer certaines caractéristiques du miroir courtois. Outre que la référence est introduite comme une comparaison ${ }^{36}$ - comme souvent dans les écrits monastiques ${ }^{37}$-, elle correspond à une vision de $l^{10 i n^{38}}$ et, surtout, à une vision partielle : chacun des cristaux ne reflète que «l'une moitié dou vergier » (v. 1562) et il n'est aucun spectateur qui puisse embrasser en un seul regard la totalité de cette "image». Précisément, la contemplation dans le speculum paulinien, seule possible ici-bas et sous le régime de la foi ${ }^{39}$, est une contemplation ex parte, selon la formule de la Vulgate ${ }^{40}$.

Plus encore, parce que l'observation à travers un miroir est médiatisée et incomplète, elle ne constitue pas sa propre fin mais contient en elle-même la promesse d'une autre vision ${ }^{41}$ - et s'inscrit de ce fait dans un parcours anagogique : «le miroir est dynamisé. L’image tend

${ }^{33}$ Marie-Madeleine Davy, Théologie et mystique de Guillaume de Saint-Thierry, I, La Connaissance de Dieu, Paris, Vrin, «Études de théologie et d'histoire de la spiritualité », 1954, p. 9 (c'est nous qui soulignons).

${ }^{34}$ Cette piste amènerait à nuancer l'équivalence qui a très souvent été établie entre les deux cristaux de la fontaine et les yeux de la Dame. C'est d'ailleurs surtout le repérage de l'intertexte lyrique qui l'autorise puisque, chez Guillaume de Lorris, il n'est aucun indice autre que le dédoublement du cristal (dédoublement qui est lui-même objet de débat, comme le rappelle Félix Lecoy dans son édition du Roman de la Rose, éd. cit., t. 1, p. 265-266) qui n'aille en ce sens. Sur cette question, voir aussi la note d'Ernest Langlois (Guillaume de Lorris, Le Roman de la Rose, Paris, Firmin Didot/Champion, «Société des anciens textes français », 1921, t. 2, p. 309).

${ }^{35}$ Einar Ma $\mathrm{r}$ Jo nsson reprend ce terme à Jurgis Baltrušaitis, Le Miroir : essai sur une légende scientifique. Révélations, science-fiction et fallacies, Paris, Seuil/Éditions Aline Elmayan, 1978.

${ }^{36}$ «Et por la chose feire entendre, / un essample vos voil aprendre : / ausi con li mireors montre / les choses qui sont a l'encontre / et i voit l'en sanz coverture / et lor color et lor figure, / tot autresi vos di por voir, / que li cristaus sanz decevoir / tot l'estre dou vergier encuse / a celui qui en l'eve muse » (v. 1551-1560).

${ }^{37}$ Ce statut analogique nous paraît un indice important du sens de l'épisode : toute vision indirecte est une vision comme à travers un miroir. Il nous semble en effet que dans le champ discursif du Moyen Âge central, cette mention signale moins la transparence ou la réflexion d'une image ressemblante que le dispositif d'une vision catoptrique, c'est-à-dire le paradigme d'un type de contemplation : la contemplation médiatisée.

${ }^{38}$ De même, de nombreux textes cisterciens soulignent cet aspect. Ainsi, dans le $26^{\mathrm{e}}$ sermon sur le Cantique des cantiques (Bernard de Clairvaux, Sermons sur le Cantique des cantiques, éd. Jean Leclercq, Henri Rochais et Charles-Henri Talbot, trad. et notes Paul Verdeyen et Raffaele Fassetta, Paris, Cerf, «Sources chrétiennes », 1998, 26, 1, t. 2, p. 278-279), Bernard construit son discours autour de plusieurs références scripturaires: la formule paulinienne, "voir dans un miroir et en énigme », est associée à la position éloignée de l'homme, "en exil loin du Seigneur », selon une formule de la seconde épître aux Corinthiens (II Cor. 5, 6).

${ }^{39}$ Guillaume de Saint-Thierry, Enigma Fidei, éd. cit., 3 sq., p. 92 sq.

${ }^{40}$ Voir par exemple Bernard de Clairvaux, Sermons sur le Cantique des cantiques, éd. cit., 55, 4, t. 4, p. 134.

${ }^{41}$ Cf. Einar Ma r Jo nsson, Le Miroir..., op. cit., p. 69. 
vers Dieu $»^{42}$. Les connecteurs temporels de l'Épître aux Corinthiens nunc/ tunc sont largement repris (avec le couple nunc / in futuro dans le passage qui suit) et ils amènent à distinguer, de manière tout à fait traditionnelle, deux types de vision, comme chez Bernard par exemple :

« Bienheureux, donc, ceux qui ont le cœur pur car ils verront Dieu » [Matth. 5, 8] : maintenant, certes, «dans un miroir, en énigme, mais plus tard face à face » [1 Cor. 13, 12] ; ce sera quand aura été achevée la purification de notre face : " alors il la fera paraître devant lui, dans sa gloire, sans tache et sans ride » [Éphés. 5, 27] $]^{43}$.

La vision indirecte est en quelque sorte l'annonce (voire la préparation) d'une autre vision, directe cette fois, facie ad faciem. La référence au miroir, dès lors, ne serait pas à considérer de manière indépendante ; elle serait le premier terme d'un schéma dynamique. Et de fait, si entre la figure de Narcisse et celle de l'Amant, la logique est celle du contre-point, c'est un autre principe qui ordonne la suite de l'épisode: cette tension entre une contemplation médiate et une contemplation face à face amène à repérer dans le Roman un jeu d'échos et un mouvement de progression. L'observation spéculaire fait naître chez le spectateur le désir d'une saisie directe de l'objet du regard :

et lors m'en prist si grant envie / que ne lessasse por Pavie / ne por Paris que je n'alasse /

la ou je vi la greignor tase. [...] / Vers les rosiers tantost me très ; / et bien sachiez, quant

je fui pres, / l'odor des roses savoree / m'entra jusques en la coree. (v. 1617-1626)

Le premier moment, donc, est à la fois une vision et, par le truchement du désir, l'appel d'une autre vision, vision de près et sans intermédiaire. Celle-ci représenterait un stade plus avancé dans la recherche de l'intimité : c'est ainsi, du moins, que Bernard explique le passage où l'épouse perçoit les parfums de son bien-aimét4. Premier volet d'un diptyque, l'épisode du miroir serait d'emblée orienté vers ce deuxième moment - et, de la sorte, il introduirait le texte dans le «régime ordinaire du récit de fiction $»^{45}$ : comme le montre Emmanuèle Baumgartner à partir de l'étude du jeu des temps dans le roman, alors que la multiplication des descriptions dans la première partie permet d'accomplir « le tour de force rhétorique » de « cern[er] et fix[er] dans l'espace du texte [...] un éternel présent du temps ${ }^{46}$,

la Fontaine d'Amour, elle, est le lieu crucial à partir duquel le récit se ressource, se relance et prend très exactement un nouveau « cours », désormais orienté, canalisé par la vision du bouton de rose ${ }^{47}$.

${ }^{42}$ Robert Javelet, Image et ressemblance au XII siècle..., op. cit., t. 1, p. 386. Et l'auteur de poursuivre : « s'il n'en est pas ainsi, [...] elle se referme vainement sur son phantasme ».

${ }^{43}$ Bernard de Clairvaux, De Conversione ad clericos, dans Bernard de Clairvaux, Le Précepte et la dispense, La conversion, éd. Jean Leclercq, Henri Rochais et Charles-Henri Talbot, trad. et notes Françoise Callerot, Jürgen Miethke et Christiane Jaquinod, Paris, Cerf, « Sources chrétiennes », 2000, 30, p. 396-397 : « Beati igitur mundo corde, quoniam ipsi Deum videbunt : nunc quidem per speculum in aenigmate, in futuro autem facie ad faciem, ubi nimirum faciei nostrae fuerit consummata mundities, ut sibi eam exhibeat gloriosam, non habentem maculam neque rugam ».

${ }^{44} C f$. Bernard de Clairvaux, Sermons sur le Cantique des cantiques, éd. cit., 22, t. 2.

${ }^{45}$ Emmanuèle Baumgartner, «Le jeu des temps ou le rêve raconté de Guillaume de Lorris », dans De l'histoire de Troie au livre du Graal. Le temps, le récit (XII -XIII siècles), Orléans, Paradigme, «Varia », 1994, p. 464.

${ }^{46}$ Ibid., p. 462.

${ }^{47}$ Ibid., p. 465. 
Sous le rapport des modalités de la vision, l'image du miroir dans le texte de Guillaume de Lorris révèle donc avant tout la nature du dispositif spéculaire installé autour de la fontaine : celui-ci permet de médiatiser la perception de l'objet d'amour et de différer sa contemplation directe. Cette configuration visuelle pourrait être comprise comme une construction qui signale l'excellence de la rose : la fleur ne peut d'abord être vue que dans un miroir ; corollairement, parce que la rencontre facie ad faciem n'est offerte qu'aux plus purs, cette station du narrateur devant la fontaine de Narcisse le conduit sur une véritable voie de perfectionnement.

\section{RÉVÉLATION - Le miroir comme espace de demontrance}

Selon notre hypothèse, examiner le miroir du Roman de la Rose à partir du discours mystique permet de percevoir un deuxième mouvement, descendant. Si l'Amant progresse de la vision médiate à la vision directe, le miroir pourrait peut-être être également considéré comme un espace de révélation.

C'est ce qu'indique le vocabulaire de la monstrance : au même titre que les cristaux (v. 1603), «li mireors montre» (v. 1553), il fait une «demontrance» (v. 1567) de toute chose. Comme le narrateur l'explique à partir de l'image du miroir, dans la vision médiatisée ce qui était caché se manifeste ${ }^{48}$ : le rosier, qui n'avait pas été mentionné dans les longs passages descriptifs qui ponctuent la promenade de l'Amant ${ }^{49}$, est désormais remarqué par le personnage $^{50}$. C'est que, si l'objet de la contemplation est bien le même lors de la pérégrination dans le jardin et lors de l'examen dans le cristal, le «niveau de perception » ou le degré de dévoilement diffère. Le narrateur rappelait, en parlant de sa déambulation :

Mes j'alai tant destre et senestre / que j'oi tot l'afere et tot l'estre / dou vergier cerchié et veü. (v. 1415-1417)

Par le filtre du miroir de la fontaine, les mêmes composantes du verger sont perçues mais, cette fois-ci, « sanz coverture » (v. 1555) :

vos di por voir / que li cristaus sanz decevoir / tot l'estre du vergier encuse / a celui qui en

l'eve muse. (v. 1557-1560)

L'écho lexical entre les deux extraits (« tot l'estre du vergier ») s'accompagne d'une variation significative. Dans le deuxième cas, plusieurs indices signalent la supériorité de ce tableau, seul capable de manifester la vérité dans toute sa transparence : "sanz coverture », "sanz decevoir », encuser. Tout se passe donc comme si le miroir, instrument d'une conversion du

${ }^{48}$ « si n'i a si petite chose, / tant soit reposte ne enclouse, / dont demontrance ne soit feite / con s'ele ert ou cristal portrete» (v. 1565-1568). On mesure bien alors, comme le note Michèle Gally, la différence avec la perception des peintures des vices : ces figures, appréhendées de manière directe, sont élucidées par une inscription et ne contiennent aucun mystère. Dans ce cas, la pause contemplative ne transforme pas l'objet vu en espace de révélation. $C f$. Michèle Gally, "Miroir d'Oiseuse, Miroir de Dieu. Théories de la vision et discours poétique dans le Roman de la Rose », dans Michèle Gally et Michel Jourde (dir.), L'Inscription du regard au Moyen Âge et à la Renaissance, Fontenay-Saint-Cloud, ENS Éditions, 1995, p. 13-35.

${ }^{49}$ Voir en particulier les v. 1321-1414.

${ }^{50}$ Voir en ce sens les remarques de Charles Méla, « Le miroir périlleux ou l'alchimie de la rose », art. cit., p. 79 sq. 
regard, était le lieu d'une épiphanie : ce dévoilement donne accès à un autre niveau de réalité. Maurice Accarie parle en ce sens de cette contemplation comme d'un «raptus vers l'essence des choses $»^{51}$.

Et de fait, si, chez les cisterciens comme chez de nombreux autres représentants de la pensée monastique, la connaissance dans le miroir reste une connaissance imparfaite, bien inférieure à la vision directe, impossible en cette vie, cette perception per speculum est toutefois elle-même plus « clairvoyante » que celle des aveugles incapables de reconnaître les traces divines dans l'œuvre de la Création ou dans les Écritures. Un développement proposé par Bernard à partir de l'image de l'ombre, qui est parfois associée à celle du miroir et qui peut se référer au même domaine, est intéressant à cet égard ${ }^{52}$. Dans le sermon 31 , il indique, nous semble-t-il, que le sens de la dialectique ombre/vérité est relatif, c'est-à-dire que cette distinction peut être appliquée à différents référents :

C'est pourquoi je pense que le Prophète a voulu signifier cela, lorsqu'il dit : « Le Christ Seigneur est Esprit devant notre face ; à son ombre nous vivons parmi les nations » [Lam. $4,20]$. En effet, «maintenant nous voyons dans un miroir, en énigme et pas encore face à face » [I Cor. 13, 12]. Mais cela, certes, tant que «nous vivrons parmi les nations »; car il en sera tout autrement parmi les anges. Alors, jouissant d'une félicité absolument identique à la leur, «nous le verrons nous aussi tel qu'il est » [I Jn 3, 2], c'est-à-dire «dans sa forme de Dieu» [Phil. 2,6], et non plus «sous le voile de l'ombre ». Nous disons donc que les hommes de l'Ancien Testament eurent en partage l'ombre et la figure, tandis que pour nous la vérité elle-même resplendit, par la grâce du Christ présent dans la chair. De même, personne ne saurait nier que, par rapport au siècle à venir, nous aussi, nous vivons encore d'une certaine manière dans l'ombre de la vérité ; à moins de contester ce que dit l'Apôtre: "Partielle est notre connaissance et partielle notre prophétie » [I Cor. 13, 9] $]^{53}$.

D'une part, on peut repérer ici plusieurs binômes qui semblent se recouvrir en partie : voir dans un miroir et en énigme / voir face à face, voir Dieu tel qu'il veut / voir Dieu tel qu'il est, voir l'ombre (et la figure) / voir la forme (ou la vérité). D'autre part et surtout, on constate que ces couples se rapportent aussi bien à la différence entre la vision des cœurs emplis de foi en ce monde et celle des bienheureux et des anges qu'à la distinction entre les Anciens, qui n’ont pu bénéficié de la Révélation, et les hommes qui ont pu connaître la présence de Dieu

${ }^{51}$ Maurice Accarie, «La vie n'est pas un songe. Le miroir brisé de Guillaume de Lorris », Théâtre, littérature et société au Moyen Âge, Nice, Serre, 2004, p. 457.

${ }^{52}$ De même, commentant différentes références scripturaires aux ombres (ombrae), Bernard indique une des interprétations possibles : « nous disons [...] que les ombres sont les figures et les énigmes des Écritures » (Bernard de Clairvaux, Sermons sur le Cantique des cantiques, éd. cit., 72, 5, t. 5, p. $122-$ 123: «dicimus umbras, figuras et aenigmata Scripturarum»); ailleurs, il explique que la contemplation de l'épouse ici-bas « se passe dans l'ombre, parce que "dans un miroir et en énigme" [I Cor. 13, 12]» (ibid., 48, 8, t. 3, p. 326-327: "At istud in umbra, quia per speculum et in aenigmate »).

${ }^{53}$ Bernard de Clairvaux, Sermons sur le Cantique des cantiques, éd. cit., 31, 8, t. 2, p. 442-445: "Unde ego puto id significatum apud Prophetam, ubi ait: Spiritus ante faciem nostram Christus Dominus; in umbra eius vivemus inter gentes: quod scilicet videamus nunc per speculum in aenigmate, et necdum facie ad faciem. At istud sane donec vivimus inter gentes; nam inter angelos aliter : quando iam indifferenti omnino felicitate cum ipsis videbimus eum et nos sicuti est, hoc est in forma Dei, et non in umbra. Quomodo namque apud veteres quidem umbram figuramque dicimus exstitisse, nobis autem per gratiam Christi in carne praesentis ipsam per se illucescere veritatem, ita nos quoque respectu futuri saeculi in quadam interim veritatis umbra vivere non negabit, nisi qui non acquiescit Apostolo dicenti : Ex parte cognoscimus et ex parte prophetamus ». 
grâce à l'Incarnation. S'agissant de l'interprétation mystique, il y a donc différents niveaux de dévoilements - et différents "degrés d'être » ${ }^{54}$. Il nous semble dès lors que si voilée qu'elle puisse être, la contemplation per speculum n'est pas seulement à envisager dans son rapport avec l'observation directe ou facie ad faciem : par rapport à l'aveuglement de celui qui ignore la Révélation, c'est déjà une demontrance.

Cette proposition de lecture, appliquée à un récit courtois, amène tout d'abord à s'interroger sur la référence au soleil dans le Roman de la Rose : alors que l'Amant accède à une vision plus transparente, dans le même temps, le texte suggère comme un geste venu d'en haut :

Quant li solaus, qui tot aguiete, / ses rais en la fontaine giete / et la clarté aval descent, / lors perent colors plus de cent/ ou cristal, qui par le soleil/devient inde, jaune et vermeil. / Si est cil cristaus merveilleus, / une tel force a que li leus, / arbres et flors, et quan qu'aorne / le vergier, i pert tot a orne. (v. 1541-1549)

On peut noter dans ce passage une constellation de termes qui rappellent le discours mystique : le soleil, traditionnellement associé au divin, est ici le sujet des verbes aguetier et getier ; la référence aux rayons, associée à la descente de la clarté, ne manque pas d'évoquer la « cascade d'illuminations $»^{55}$ qu'on trouve chez Hugues de Saint-Victor par exemple. On peut peut-être aussi s'interroger sur la rime : aorne la orne ${ }^{56}$. Ne faudrait-il pas, derrière cette association entre l'ornatus et l'ordo pour la description d'un lieu paradisiaque, entendre comme des vestiges du discours des spirituels ? Quoi qu'il en soit, tous ces indices pourraient être compris comme autant d'éléments qui construisent le spectacle du miroir comme un don offert à l'Amant. En d'autres termes, la cause de la vision du spectateur, ce serait cette descente de lumière ; le rapport temporel entre les deux membres de la phrase est aussi un rapport de cause à effet : "Quant li solaus [...] / ses rais en la fontaine giete / et la clarté aval descent, / lors perent colors plus de cent ». Cette interprétation est corroborée par le lexique de la merveille, auquel il faudrait donner tout son sens : le cristal est un objet « merveilleus » (v. 1546), la description du double phénomène d'illumination et de «coloration » est ellemême une merveille $e^{57}$ et sans doute n'est-ce pas un hasard si le pouvoir de la pierre est décrit comme une «force et [une] vertuz» $(\text { v. 1609) })^{58}$. Au fond, ce n'est que par l'action

\footnotetext{
${ }^{54}$ Einar Ma r Jo nsson, Le Miroir..., op. cit., p. 93.

${ }^{55}$ Roger Baron, Études sur Hugues de Saint-Victor, Paris, Desclée de Brouwer, 1963, p. 154.

${ }^{56}$ Elle apparaît également dans les éditions d'Ernest Langlois (éd. cit., t. 2, v. 1551-1552) et d'Armand Strubel (Guillaume de Lorris et Jean de Meun, Le Roman de la Rose, d'après les manuscrits BN 12786 et BN 378, Paris, Librairie générale française, «Le Livre de Poche. Lettres gothiques », 1992). Nous avons déjà évoqué ce passage dans La Question de la beauté. Modes d'écriture et modes de pensée dans le discours romanesque du début du XIII siècle, à paraître aux éditions Champion.

${ }^{57}$ « Mes une chose vos dirai / qu'a merveille, ce cuit, tendroiz / maintenant que vos l'entendroiz » (v. 1538-1540). Selon nous, il faut voir dans ce passage plus qu'une référence rhétorique à la merveille dans le cadre d'une captatio benevolentiae: le substantif annonce le caractère proprement extraordinaire du phénomène lumineux.

${ }^{58}$ Pour ces termes, voir les v. 1547 et 1608 de l'éd. cit. d'Armand Strubel. On ne serait pas très loin, semble-t-il, des merveilles miraculeuses telles qu'elles sont définies par Jean-René Valette, La Pensée $d u$ Graal. Fiction littéraire et théologie (XII ${ }^{e}$ XIII ${ }^{e}$ siècle), Paris, Champion, « Nouvelle bibliothèque du Moyen Âge », 2008, p. 230.
} 
« surnaturelle» de cette illumination venue d'en haut que le spectateur accède à la vision, médiatisée, d'un spectacle dont la beauté, l'ordonnancement et la clarté sont inédits. Le dispositif qui, dans le texte, associe le soleil et le miroir, ferait ainsi de la contemplation du verger le résultat d'une révélation (non pas divine, mais proche de celle qui est décrite dans le discours mystique): c'est bien une représentation nouvelle, plus belle que tout ce qui précédait, qui est offerte au regard de l'Amant.

Voir le miroir de la fontaine comme l'espace d'une demontrance n'attire pas seulement l'attention sur le rôle du soleil ; cette proposition amène également à s'interroger sur le rapport entre le sujet regardant et l'objet contemplé. Que, grâce aux cristaux, la représentation du verger ressortisse à une manifestation paraît avant tout signaler, au même titre que la vision indirecte, ce qu'on pourrait appeler la « dissemblance » entre le personnage et le rosier qu'il observe. Distinguant la relation entre le Père et le Fils de la relation entre l'homme et Dieu, Bernard de Clairvaux prend l'exemple de Paul et explique :

Paul est grand, certes ; mais, si haut qu'il tende sa bouche, s'élèverait-il même jusqu'au troisième ciel, il faut bien pourtant qu'il demeure en deçà de la bouche du Très-Haut, et qu'il se tienne dans ses limites. Et comme il est incapable d'atteindre au visage de gloire [II Cor. 3, 7], qu'il demande humblement à Dieu de descendre à son niveau et de lui envoyer d'en haut le baiser ${ }^{59}$.

Vision à travers un miroir, vision déterminée par un don : cette construction, dans le roman, pourrait une nouvelle fois être comprise comme une manière de signifier l'excellence de ce qui est vu. Ces éléments, qui font sens dans le discours mystique, pourraient alors être considérés comme des unités sémantiques «disponibles » pour dire l'inestimable valeur de cette vision courtoise. Ce qui importerait, c'est la connotation dont est porteuse dans un autre champ discursif l'association du soleil, des rayons, du miroir, etc. La conjonction de tous ces traits ou de tous de ces marqueurs d'excellence, au fond, dirait que la contemplation à laquelle l'Amant accède est des plus hautes.

Si l'on s'en tient au seul parcours de l'Amant et sous le rapport de cette hiérarchisation des modes de perception, trois étapes peuvent donc être distinguées :

- la «vision aveugle» du personnage lors de la promenade (la rose reste invisible pour lui);

- la vision médiatisée dans la contemplation per speculum, à travers le miroir de la fontaine ;

- la vision facie ad faciem, contemplation différée que de nouveaux obstacles repoussent sans cesse ${ }^{60}$.

\section{AMOUR - Le miroir comme « Fontaine d'Amors »}

${ }^{59}$ Bernard de Clairvaux, Sermons sur le Cantique des cantiques, éd. cit., 8, 8, t. 1, p. 190-191: «Magnus quidem Paulus; sed quantumlibet sursum porrigat os, etiamsi se usque ad tertium caelum extendat, citra os Altissimi tamen necesse est ut remaneat, et modo suo contentus in se subsistat, et cum pertingere ad vultum gloriae non valebit, condescendi sibi et ex alto transmitti osculum humiliter petat».

${ }^{60}$ Voir infra, note 66. 
Nous voudrions, pour terminer, voir en quoi cette fontaine est aussi, plus encore qu'une étape essentielle dans le cheminement du personnage et dans le déroulement du récit, un lieu de fixation de l'objet du désir et le moment d'une définition de cette haute amor qu'est la fin'amor. Nous faisons en effet l'hypothèse que ce qui est en jeu dans la conversion du regard du personnage, c'est la découverte du bien aimer. Cette relation entre la vision dans un miroir et l'amour n'est pas une spécificité du discours mystique : on la trouve déjà chez Ovide et elle caractérise également la physique de l'amour de la lyrique courtoise. Simplement, selon nous, cette conction entre vision médiate, révélation et amour peut aussi trouver une cohérence à partir du texte paulinien et de ses interprétations cisterciennes ${ }^{61}$.

De fait, dans le roman, le dispositif spéculaire qui a été décrit reçoit plusieurs noms dont celui de « Fontaine d'Amors » (v. 1595) :

fu ceste fontaine apelee/ la Fontaine d'Amors par droit, / dont plusor on en maint endroit / parlé en romanz et en livre. (v. 1594-1597)

La double référence à des textes de langue romane et à des livres, en latin ${ }^{62}$, contribuerait à autoriser ce rapprochement avec le «fons amoris» ou "fons caritatis ». Comme le note d'ailleurs Emmanuèle Baumgartner, dans le Roman de la Rose, la fontaine de mort est devenue fontaine de vie ${ }^{63}$. Tandis que plusieurs réécritures soulignent l'immobilité des eaux qui reflètent l'image de Narcisse, le texte de Guillaume de Lorris insiste sur l'abondance des flots et sur leur tendance à irriguer tout ce qui les entoure ${ }^{64}$ :

L'eve est tot jorz fresche et novele, / qui nuit et jor sort a grant ondes / par .II. doiz cleres et parfondes. / Tot entor croist l'erbe menue, / qui vient por l'eve espesse et drue / ne en yver ne puet mourir, / ne l'eve sechier ne tarir. (v. 1528-1534)

De telles notations sont fréquentes pour évoquer, par une image, la diffusion de la charité. De là, l'équivalence posée par le texte romanesque entre la fontaine-miroir et la fontaine d'amour peut-elle être éclairée par le sens que la mystique donne au speculum ?

La vision parfaite de Dieu, explique Bernard dans le sermon 82, correspond au moment où l'âme aura recouvré la ressemblance parfaite : entre le divin et l'humain, il y aura alors une contemplation claire, " une connaissance plénière », une unité d'esprit. Cette vision, en d'autres termes, scellera leur « affection chaste et accomplie » et l'accomplissement de leur amour $^{65}$. De même, chez Isaac de l'Étoile, la rencontre face à face est à la fois une contemplation de Dieu et une délectation en Dieu ${ }^{66}$. La corrélation entre l'amour et la dialectique vision directe / vision indirecte contenue dans la formule paulinienne concerne

${ }^{61} \mathrm{Au}$ demeurant, l'association entre speculum et caritas est déjà au centre de l'Épître de Paul : voir I Cor. 13. Sur ce point, $c f$. Einar Ma r Jo nsson, Le Miroir ..., op. cit., p. 68 sq.

${ }^{62}$ Voir notamment Emmanuèle Baumgartner, "Le livre et le roman (XII-XIII ${ }^{\mathrm{e}}$ siècles) », dans De l'histoire de Troie au livre du Graal, op. cit., p. 37-38.

${ }^{63}$ Emmanuèle Baumgartner, « Narcisse à la fontaine : du "conte" à l’"exemple" », art. cit.

${ }^{64}$ Ibid.

${ }^{65}$ Bernard de Clairvaux, Sermons sur le Cantique des cantiques, éd. cit., 82, 8, t. 5, p. 338-339.

${ }^{66}$ Isaac de 1'Étoile, Sermons, éd. et trad. Anselm Hoste, Gaston Salet, Gaetano Raciti, Paris, Cerf, « Sources chrétiennes », 1974, 25, 4-15, t. 2, p. 118-127. 
ainsi avant tout l'aboutissement du parcours mystique : celui-ci consiste en une contemplation qui offre l'expérience de la joie suprême ${ }^{67}$.

L'articulation entre voir et aimer, toutefois, n'intervient pas seulement au moment de l'union; la disposition affective du « spectateur » agit aussi pour ainsi dire en amont, comme une condition de la rencontre. Si, comme le rappelle Guillaume de Saint-Thierry dans le Speculum fidei, la saisie facie ad faciem offre la perfection de l'amour, la «magnitudo amoris $»^{68}$, la perception médiatisée de Dieu (dans un miroir) exige déjà la charité ${ }^{69}$. En effet, cette perfection, dans la pensée monastique, résulte de la progression de l'âme dans sa quête de la ressemblance perdue : pour l'épouse, qui a été cherchée avant de chercher ${ }^{70}$, il convient de vouloir ce que Dieu veut et d'ordonner en elle la charité ; il s'agit de passer d'un amour esclave exclusivement orienté vers soi-même à un amour qui, selon le mot de Guillaume, ne convoite rien « en dehors de Dieu », « extra Deum $»^{71}$.

Proposer une lecture de l'épisode romanesque de la fontaine à partir de ces pistes, rappelées à grands traits, ne consistera pas dans la recherche d'une transposition de la pensée mystique. Simplement, ces éléments qui participent, pendant le Moyen Âge central, de la construction d'un langage de l'amour ${ }^{72}$ doté d'une haute valeur, peuvent aider à établir une grille d'investigation pour l'étude du discours courtois; en d'autres termes, ils peuvent permettre de repérer les «questions » auxquelles s'efforcent de répondre, de manières différentes, des textes divers, composés dans des langues diverses.

${ }^{67} \mathrm{Si}$, dans le cadre de cette étude, il n'est pas possible de développer cette piste, signalons toutefois que, dans le roman, l'indexation de la jouissance amoureuse à la rencontre ultime espérée, proche de la description des mystiques, participe certainement de la valorisation de cette érotique profane, détachée du charnel. Il faut toutefois signaler une différence importante : contrairement au parcours noétique et affectif mis en place par les spirituels à partir de la distinction paulinienne, la seule vision directe n'est pas l'aboutissement de l'amour dans le Roman. Le récit de Guillaume de Lorris multiplie les stratégies dilatoires, que celles-ci ressortissent à des dispositifs visuels (après la vision médiatisée des rosiers « en un destor », v. 1615, vision directe d'une rose, v. 1653-1654, puis vision « de [...] pres », v. 2808, mais l'objet désiré reste encore en partie dissimulé : «el n'iere pas si overte/que la graine fust descovierte ; / ençois estoit encor enclose/ entre les fueilles de la rose », v. 3345-3348) ou à des obstacles empêchant tout contact (les chardons par exemple ou encore les blessures infligées par les flèches d'Amour qui empêchent le personnage de s'approcher, les opposants, etc.). La possession de la rose est sans cesse différée - et jamais actualisée en raison, notamment, de l'inachèvement de l'œuvre de Guillaume.

${ }^{68}$ Guillaume de Saint-Thierry, Speculum fidei, dans Guillaume de Saint-Thierry, Deux traités sur la foi. Le Miroir de la foi, l'Énigme de la foi, op. cit., 26, p. 46.

${ }^{69}$ Ibid., 1-6, p. 24-31.

${ }^{70}$ Cf. ibid., 78, p. 88-91.

${ }^{71}$ Ibid., 8, p. 32-33. Sur ces questions, voir aussi chez Bernard, par exemple, le De diligendo Deo et les quatre degrés de l'amour de Dieu, dans Bernard de Clairvaux, L'Amour de Dieu, La Grâce et le libre-arbitre, éd. et trad. Jean Leclercq, Françoise Callerot, Jean Christophe, Paris, Cerf, «Sources chrétiennes », 1993, 23-29, p. 116-137.

${ }^{72}$ Nous reprenons cette formule au titre français de John W. Baldwin, Les Langages de l'amour dans la France de Philippe Auguste. La sexualité dans la France du Nord au tournant du XII siècle, trad. Béatrice Bonne, Paris, Fayard, 1997 [1994]. 
Chez Guillaume de Lorris où les différents modes de contemplation correspondent aussi aux progrès de l'amour, tout commence par le don de la première vision autour de la « Fontaine d'Amors ». Avant cette demontrance, le personnage n'est pas encore un amant ou un amoureux - il se distingue tout au plus par sa disponibilité à aimer. C'est la vision dans la fontaine-miroir qui ferait naître en lui l'amour et le désir de chercher ce qui lui a déjà été manifesté dans un reflet. On pourrait alors envisager le trajet du protagoniste jusqu'à la vision directe du rosier comme une réponse à cet appel qu'a constitué la première contemplation. D'ailleurs, immédiatement après la description de la fontaine et de ses cristaux, dans un développement sur les dangers de l'amour, le narrateur indique :

ici [dans ce miroir] se changent li corage, / ci n'a mestier sens ne mesure ${ }^{73}, /$ ci est d'amer volenté pure. (v. 1582-1584)

Ne pourrait-on dès lors voir le miroir comme un lieu de conversion affective, un lieu où « se changent li corage » afin de devenir « volenté pure » «d'amer » ? Significativement, ce n'est qu'après cette pause, assortie d'une forte tension scopique, que le personnage, en plusieurs étapes, fixe son désir sur un des boutons - comme s'il était nécessaire que, préalablement, le regard fût correctement orienté et que le cœur fût entièrement préparé à l'amour :

El miroër entre mil choses / choisi rosiers chargiez de roses / qui estoient en un destor, / d'une haie bien clos entor. (v. 1613-1616)

Entre les autres en eslui / un si tres bel, envers celui / nul des autres rien ne prisé / puis que celui bien avisé ${ }^{74}$. (v. 1653-1656)

Selon notre hypothèse, l'épisode de la fontaine, stase dans le récit qui assure la relance narrative, raconte aussi, avant même le jet de la flèche du dieu, les commencements de l'amour $^{75}$ : il décrit les progrès du personnage dans l'identification de l'objet du désir.

C'est alors sous le double rapport de la contemplation et de l'amor que Narcisse et l'Amant peuvent être comparés. Face au héros du roman, la figure ovidienne, qui regarde pourtant dans le même miroir, offre l' « essample » d'un mauvais spectateur et d'un mauvais amant - comme si l'absence de bonnes dispositions affectives interdisait l'accès à la vision per speculum du plus haut des objets d'amour. Nous présenterons ici quelques suggestions destinées à souligner les jeux d'inversion entre les deux personnages ${ }^{76}$.

${ }^{73} C f$. Bernard de Clairvaux, De diligendo Deo, éd. cit., 1, p. 60-61 : « la cause de notre amour de Dieu, c'est Dieu même; la mesure, c'est de l'aimer sans mesure » («causa diligendi Deum, Deus est; modus, sine modo diligere $\gg)$.

${ }^{74}$ Sur le choix du bouton, voir aussi Maurice Accarie, «La vie n'est pas un songe. Le miroir brisé de Guillaume de Lorris », art. cit., p. 454 sq.

${ }^{75}$ Sur la double nécessité de la «précédence » de la grâce et de la bonne volonté dans les progrès de l'amour, voir par exemple Guillaume de Saint-Thierry qui rappelle que l'homme ne peut aimer que si, prévenu par la grâce, il possède une bonne volonté : "Commence à aimer, c'est-à-dire agis en sorte que tu veuilles et tu commenceras à croire ; tu croiras dans la mesure où tu voudras, c'est-à-dire dans la mesure où tu aimeras. La volonté en effet est le commencement de l'amour. L'amour est une volonté ardente » (Guillaume de Saint-Thierry, Speculum fidei, éd. cit., 12, p. 36-37 (nous soulignons): "Incipe diligere, hoc est, age ut velis; et incipies credere; et tantum credes, quantum voles, hoc est, quantum diliges. Voluntas enim initium amoris est. Amor siquidem vehemens voluntas est »).

${ }^{76}$ Pour une autre comparaison, fort intéressante, entre les deux personnages, $c f$. Jean Wirth, « La fontaine de Narcisse dans le Roman de la Rose », dans Leïla El-Wakil, Stéphanie Pallini, Lada Umstätter-Mamedova (dir.), Études transversales. Mélanges en l'honneur de Pierre Vaisse, Lyon, Presses universitaires de Lyon, 2005, p. 11-24. 
- Intériorité des spectateurs :

\begin{tabular}{|c|l|l|}
\hline & \multicolumn{1}{|c|}{ Narcisse } & \multicolumn{1}{c|}{ L’Amant } \\
\hline $\begin{array}{c}\text { Motif de la pause } \\
\text { près de la fontaine }\end{array}$ & Désir de se désaltérer (v. 1470-1480) & $\begin{array}{l}\text { Désir prudent de contempler la } \\
\text { fontaine (v. 1517-1525)7 }\end{array}$ \\
\hline $\begin{array}{c}\text { «Qualité » de la } \\
\text { volonté }\end{array}$ & «d'amer si lache» (v. 1458) & "d'amer volenté pure » (v. 1584) \\
\hline
\end{tabular}

- Caractéristiques de ce qui est vu :

\begin{tabular}{|c|c|c|}
\hline & Narcisse & L'Amant \\
\hline $\begin{array}{l}\text { Lieu d'apparition } \\
\text { (en raison de } \\
\text { l'orientation du } \\
\text { regard des } \\
\text { spectateurs) }\end{array}$ & $\begin{array}{l}\text { Regard qui reste à la surface de la } \\
\text { fontaine (et ne peut donc percevoir le } \\
\text { cristal) }\end{array}$ & $\begin{array}{l}\text { Regard qui scrute attentivement le } \\
\text { «fonz de la fontaine } »(v .1535-1537) \text {. }\end{array}$ \\
\hline Nature du reflet & $\begin{array}{l}\text { L'objet contemplé par Narcisse est } \\
\text { défini comme une ombre } \text { (v. } 1484 \text { et }^{80} \text { 1492), un « reflet», qui est, ici, un } \\
\text { « substitut fallacieux de l'être » }{ }^{81} \text {. }\end{array}$ & $\begin{array}{l}\text { Miroir donnant accès à un niveau de } \\
\text { réalité supérieur }{ }^{82} \text {. }\end{array}$ \\
\hline Objet vu & $\begin{array}{l}\text { Personnage masculin (« la figure / } \\
\text { d'un esfant bel a desmesure », } \\
\text { v. 1485-1486), qui n'est pas identifié } \\
\text { par le spectateur comme son propre } \\
\text { reflet }^{83} \text {. }\end{array}$ & $\begin{array}{l}\text { Rosiers au sein desquels une rose se } \\
\text { détachera }^{84} \text {. }\end{array}$ \\
\hline
\end{tabular}

- Caractéristiques de l'amour :

\begin{tabular}{|c|c|c|}
\hline & Narcisse & L'Amant \\
\hline Objet aimé & $\begin{array}{l}\text { Narcisse " ama son ombre demainne » } \\
\text { (v. 1492): non seulement Narcisse ne } \\
\text { perçoit pas que l'objet aimé est une } \\
\text { représentation de lui-même mais, en } \\
\text { outre et surtout, comme la syntaxe le }\end{array}$ & $\begin{array}{l}\text { Dissociation entre l'image perçue } \\
\text { dans le miroir (rosier) et l'objet aimé } \\
\text { (rose). }\end{array}$ \\
\hline
\end{tabular}

77 À partir de cette opposition, on peut peut-être penser aux spirituels qui, dans l'apprentissage de l'amour, distinguent l'amour de soi (et la recherche de son intérêt) et l'amour de Dieu pour lui-même. Voir par exemple Bernard de Clairvaux, De diligendo Deo, éd. cit.

${ }^{78}$ Cf. Jean Wirth, « La fontaine de Narcisse dans le Roman de la Rose », art. cit., p. 18.

${ }^{79}$ «La perception de l'aspect (species) extérieur des créatures [qui sont souvent comparées à des miroirs chez les spirituels] est loin d'atteindre à la qualité intérieure. Que l'appréhension de cet aspect, de cette "species ", soit de peu d'importance en elle-même - elle n'est que la surface des choses et parfois devient source d'erreur - il n'empêche qu'elle est normalement indispensable pour la connaissance. Il ne faut pas qu'elle soit écran ou ombre vaine, mais elle doit être médiatrice de vérité. Elle l'est si l'esprit ne cherche pas au dehors, s'il pénètre jusqu'au signe qu'elle révèle » (Robert Javelet, Image et ressemblance au XII siècle..., op. cit., t. 1, p. 85, nous soulignons).

${ }^{80} \mathrm{Si}$ chez Bernard par exemple le terme umbra peut aussi désigner le reflet perçu dans la vision per speculum, il semble que dans le texte de langue romane le substantif ombre se rapporte exclusivement à cette image trompeuse perçue par Narcisse (voir en ce sens le glossaire établi par Félix Lecoy, éd. cit., t. 3, p. 250).

${ }^{81}$ Armand Strubel, La Rose, Renart et le Graal. La littérature allégorique en France au XIII siècle, Paris, Champion, « Nouvelle bibliothèque du Moyen Âge », 1989, p. 208.

${ }^{82} \mathrm{C}$ 'est ce que nous avons essayé de montrer supra.

${ }^{83}$ Rappelons que dans la tradition du symbolisme catoptrique, le miroir est aussi un instrument de la connaissance de soi. $C f$. Einar Ma r Jo nsson, Le Miroir..., op. cit.

${ }^{84}$ Sur la différence entre masculin et féminin (« c'est cele qui tant a de pris / et tant est digne d'estre amee / qu'el doit estre Rose clamee », v. 42-44), voir la proposition de Jean Wirth, « La fontaine de Narcisse dans le Roman de la Rose », art. cit. 


\begin{tabular}{|c|c|c|}
\hline & $\begin{array}{l}\text { montre, il aime l'ombre et non ce } \\
\text { qu'elle représente }^{85} \text {. }\end{array}$ & \\
\hline Nature de l'amour & $\begin{array}{l}\text { C'est, nous semble-t-il, la question de } \\
\text { la réciprocité amoureuse qui donne } \\
\text { une cohérence à toute la réécriture du } \\
\text { récit ovidien. Coupable de ne pas } \\
\text { rendre à Écho son affection (v. 1442- } \\
\text { 1450), Narcisse est puni par où il a } \\
\text { péché (il est " destroiz d'autretele } \\
\text { amor », v. 1460) : il s'éprend d'une } \\
\text { ombre, qui ne peut l'aimer à son tour. } \\
\text { C'est donc logiquement sur un appel à } \\
\text { répondre à l'amour que se clôt la } \\
\text { digression: «Dames, cest essample } \\
\text { aprenez, / qui vers vos amis } \\
\text { mesprenez » (v. 1505-1506). L'amour } \\
\text { de Narcisse pour son ombre, qui } \\
\text { repose sur une illusion, ne peut donc, } \\
\text { par définition, être réciproque. }\end{array}$ & $\begin{array}{l}\text { Aussi lointain que soit l'objet aimé, } \\
\text { aussi nombreux que soient les } \\
\text { obstacles qui le séparent de l'Amant, } \\
\text { ce dernier multiplie les efforts pour se } \\
\text { rapprocher de la rose et pour « de la } \\
\text { belle avoir [s]es aviaus » (v. } 2566)^{86} \text {. }\end{array}$ \\
\hline $\begin{array}{l}\text { Issue de la } \\
\text { rencontre }\end{array}$ & Chute $^{87}$ et mort. & Élévation $^{88}$ et récit. \\
\hline
\end{tabular}

Pour celui qui rencontre la rose, on le voit, l'histoire de celui qui fut métamorphosé en narcisse est riche d'enseignements. Face à cette mauvaise contemplation et, partant, cette mauvese amor, la pause de l'Amant peut être envisagée comme une illustration exemplaire de la fin'amor, cette affection qui peut être réciproque mais qui, paradoxalement, maintient la tension du désir en se portant sur une figure dotée d'une éminence qui la rend quasi inaccessible. Le miroir de la fontaine, par le regard clairvoyant de ce spectateur, devient une fontaine d'amour, à laquelle on s'abreuve sans pouvoir jamais être rassasié.

À partir de ces trois pôles que constituent la vision, la révélation et l'amour, il apparaîtrait donc que la confrontation avec la lecture cistercienne de la formule paulinienne donne une relative cohérence au miroir du Roman de la Rose. Cette proposition de lecture, bien entendu, n'exclut aucun des intertextes, notamment antique et lyrique, qui ont été repérés pour le récit attribué à Guillaume de Lorris. Elle ne veut pas non plus établir une relation spécifique entre le texte courtois et l'école de charité de Cîteaux, envisagée ici comme un

${ }^{85}$ « Le Moyen Âge considère que la malheureuse aventure de Narcisse se caractérise non point par le fait qu'il s'aime lui-même [...], mais par le fait qu'il aime une image », si bien qu'on pourrait parler d'une forme d'«idolâtrie » (Giorgio Agamben, Stanze..., op. cit., p. 138). En cela, le personnage ovidien, selon Giorgio Agamben, peut être considéré comme un représentant de la fol'amor : il « brise le cercle fantasmatique en tentant de s'approprier l'image comme s'il s'agissait d'une créature bien réelle » (ibid., p. 139).

${ }^{86}$ De même, dans la mystique cistercienne, la réciprocité, inhérente à l'amour («Deus prior dilexit nos », I Jn 4, 19), est associée au caractère essentiellement inaccessible de Dieu pour l'homme.

${ }^{87} \mathrm{Ce}$ mouvement descendant n'est pas explicitement évoqué par le texte.

${ }^{88}$ C'est par l'élévation « spirituelle » du personnage, notamment grâce aux différents adjuvants, que celui-ci se rapproche toujours davantage de l'objet de son amour. 
exemple des discours disponibles sur une manière élevée d'aimer. Selon cette perspective, les rapprochements avec le roman courtois nous semblent féconds : l'objet aimé est si grand qu'il ne peut être d'emblée perçu directement; cette affection exige de la part de l'Amant une purification de sa volonté, la capacité d'aimer sans mesure ainsi qu'un regard capable de se fixer non sur une ombre mais sur la figure que le reflet ne fait que représenter; cette rencontre, enfin, est de celles qui grandissent le sujet affectif - ne serait-ce que parce qu'elle ouvre pour lui une nouvelle dimension du réel.

Cette confrontation entre deux discours invite dès lors à s'interroger sur la pertinence de la notion de "mystique courtoise » et sur la signification qui peut être donnée à cette expression $^{89}$. Si des relations de différentes natures ont déjà été établies entre les textes spirituels et les textes courtois ${ }^{90}$ et si les œuvres de langue romane accréditent elles-mêmes le rapprochement ${ }^{91}$, ne faut-il pas, plutôt que de s'interroger en termes d'influence ou de transposition ${ }^{92}$, prendre acte de cette parenté et considérer qu'elle permet à un roman comme le Roman de la Rose de construire un autre modèle amoureux, tout aussi haut en valeur, mais à destination d'une autre communauté que la communauté monastique ${ }^{93}$ ? C'est au fond l'analyse d'Erich Köhler lorsqu'il compare le récit de Guillaume de Lorris à d'autres textes de la fin'amor :

${ }^{89}$ Edmond Faral, par exemple, parlait du «mysticisme courtois » de Guillaume de Lorris (Edmond Faral, «Le Roman de la Rose et la pensée française au XIII ${ }^{\mathrm{e}}$ siècle », art. cit., p. 437). Plus récemment, l'expression de "mystique courtoise » a surtout été employée dans un autre sens : elle désigne le discours amoureux de certaines béguines rattachées à un "new movement both religious and literary, an ars subtilior of the spirit, a dolce stil religioso in which the monastic discourse on love converged with the courtly" (Barbara Newman, From Virile Woman to WomanChrist. Studies in Medieval Religion and Literature, Philadelphia, University of Pennsylvania Press, "Middle Ages Series », 1995, p. 138). Pour le domaine français, l'un des exemples les plus intéressants est celui de Marguerite Porete : sur cet auteur et son rapport avec la topique courtoise, outre la référence précédente, voir JeanRené Valette: "Marguerite Porete et la confrontation des discours : le prologue du Mirouer des Simples Ames », Cahiers de Recherches Médiévales et Humanistes, 23, 2012, p. 273-289; " Marguerite Porete et le discours courtois », dans Sean Field, Robert E. Lerner, Elsa Marmursztejn et Sylvain Piron (dir.), Marguerite Porete (1310-2010), colloque international organisé à l'occasion du septième centenaire de sa condamnation et de son exécution en place de Grève (EHESS, Paris 31 mai-

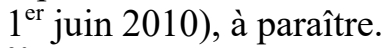

${ }^{90}$ Sur la question des relations entre l'amour courtois et l'amour de Dieu, voir notamment les études fondamentales d'Étienne Gilson, «Saint Bernard et l'amour courtois », La Théologie mystique de Saint Bernard, op. cit., p. 193-215, de Myrrha Lot-Borodine, De l'amour profane à l'amour sacré. Études de psychologie sentimentale au Moyen Âge, Paris, Nizet, 1961, de Robert Javelet, «L'amour spirituel face à l'amour courtois », dans Maurice de Gandillac et Édouard Jeanneau (dir.), Entretiens sur la Renaissance du XII siècle, Paris/La Haye, Mouton, 1968, p. 309-346 et de Charles Baladier, Érôs au Moyen Âge. Amour, désir et délectation morose, Paris, Cerf, « Histoire », 1999.

${ }^{91}$ Pour ne donner qu'un exemple, rappelons que, chez Guillaume de Lorris, Amour parle de l'« amor dou haut seintuaire » (v. 2522).

${ }^{92}$ Voir en ce sens la formule fameuse de Jean Frappier qui définit la fin'amor comme « une religion de l'amour, avec des calques nombreux de la vie spirituelle, de ses élans et de ses émotions " (Jean Frappier, "Le thème de la lumière de la Chanson de Roland au Roman de la Rose », Cahiers de l'Association internationale des études françaises, 1968, $\mathrm{n}^{\circ} 20$, p. 118).

${ }^{93} \mathrm{Cf}$. Jean Batany, «Miniature, allégorie, idéologie : "Oiseuse" et la mystique monacale récupérée par la "classe de loisir" », dans Jean Dufournet (dir.), Études sur le Roman de la Rose de Guillaume de Lorris, Paris, Champion, «Unichamp », 1984, p. 7-36. 
C'est pour la dernière fois que, dans ce paradis terrestre, la chevalerie courtoise occupe le rang suprême, au prix il est vrai, d'un renoncement à toute fonction politique, militaire, sociale, en un mot, à toute fonction historique. [...]. Le monde idéal de celle-ci [la chevalerie] est devenu, de manière définitive, un monde qui se situe en dehors du temps historique.

L'univers chevaleresque idéal a perdu toute chance de réalisation historique; c'est justement à cause de cela qu'il connaît sa plus haute spiritualisation profane, bien entendu dans une poésie du rêve, dans la révélation d'une réalité supérieure, qui, paradoxalement, renonce a priori à toute réalisation ${ }^{94}$.

En cela, si l'on peut parler du Roman de la Rose comme d'un haut lieu de la mystique courtoise, il ne faut pas comprendre que cette mystique mène à Dieu ${ }^{95}$ comme elle le fera chez Marguerite Porete par exemple ${ }^{96}$; ce roman aurait recours à un montage discursif et à des stratégies d'écriture qui, comme la contemplation à travers le miroir, peuvent historiquement signifier le plus haut degré de perfection amoureuse. En d'autres termes, ce serait non au plan du sens mais à celui des discours et des modalités pour construire de la valeur que le parallèle pourrait aussi avoir de l'intérêt.

Marie-Pascale Halary Université Lumière Lyon 2 - CIHAM

\footnotetext{
${ }^{94}$ Erich Köhler, « Narcisse, la Fontaine d'Amour et Guillaume de Lorris », art. cit., p. 102.

${ }^{95}$ Sur cette lecture du Roman de la Rose, voir Georgette Kamenetz, « La promenade d'Amant comme expérience mystique », dans Jean Dufournet (dir.), Études sur le Roman de la Rose de Guillaume de Lorris, op. cit., p. 83-104.

${ }^{96}$ Voir notamment, pour l'articulation avec l'image du miroir, Catherine M. Müller, Marguerite Porete et Marguerite d'Oingt. De l'autre côté du miroir, New York/Bern/Frankfurt am Main, P. Lang, «Currents in comparative romance. Languages and literatures », 1999, p. 118 sq.
} 